\title{
Weierstrass Representation, Degree and Classes of the Surfaces in the Four Dimensional Euclidean Space
}

\author{
Erhan Güler ${ }^{*}$, Ömer Kişi² \\ ${ }^{1}$ Bartın University, Faculty of Science, Department of Mathematics, 74100 Bartın, Turkey \\ ergler@gmail.com, eguler@bartin.edu.tr \\ ${ }^{2}$ Bartın University, Faculty of Science, Department of Mathematics, 74100 Bartın, Turkey \\ okisi@bartin.edu.tr \\ ${ }^{*}$ Corresponding author \\ Geliş / Recieved: $8^{\text {th }}$ September 2016 \\ Kabul / Accepted: 14 $4^{\text {th }}$ December 2016 \\ DOI: http://dx.doi.org/10.18466/cbujos.302660
}

\begin{abstract}
We study two parameters families of Bour-type and Enneper-type minimal surfaces using the Weierstrass representation in the four dimensional Euclidean space. We obtain implicit algebraic equations, degree and classes of the surfaces.
\end{abstract}

Keywords - 4-space, surface, Weierstrass representation, degree, class.

This work has supported by Bartın University-Scientific Research Projects Commision-(Project Number: BAP 2016-FEN-A-006).

\section{Surfaces in $\mathbb{R}^{4}$}

In Moore [4], we find a general definition of rotation surfaces in $\mathbb{R}^{4}$ :

$$
\begin{aligned}
X(u, t)= & \left(x_{1}(u) \cos (a t)-x_{2}(u) \sin (a t),\right. \\
& x_{1}(u) \cos (a t)+x_{2}(u) \sin (a t), \\
& x_{3}(u) \cos (b t)-x_{4}(u) \sin (b t), \\
& \left.x_{3}(u) \cos (b t)+x_{4}(u) \sin (b t)\right) .
\end{aligned}
$$

We propose that we look at a restricted case of this, found in Ganchev-Milousheva [2] :

$$
W(u, t)=\left(x_{1}(u), x_{2}(u), r(u) \cos (t), r(u) \sin (t)\right) .
$$

The first we think is a bit too general since the curve is not located in any subspace before rotation.

At any rate this has:

$$
g(\partial u, \partial u)=r^{\prime 2}+\left(x_{1}\right)^{\prime 2}+\left(x_{2}\right)^{\prime 2}=1
$$

if we use arc length parametrization, $g(\partial u, \partial t)=0$ and $g(\partial t, \partial t)=r^{2}$

Using the Weierstrass representation in Section 2, we give two parameters familes of Bour's-type (in Section 3) and Enneper's-type (in Section 4) minimal surfaces in the four dimensional Euclidean space. We also calculate implicit algebraic equations of the surfaces, degrees and classes of the surfaces.

\section{Weierstrass equations for a minimal surface}

$$
\text { in } \mathbb{R}^{4}
$$

In Hoffman and Osserman [3] , p.45, they give the Weierstrass equations for a minimal surface in $\mathbb{R}^{4}$ : 
CBÜ F Bil. Dergi., Cilt 13, Sayı 1, 2017, 155-163 s

$$
\Phi(z)=\frac{\psi}{2}(1+f g, i(1-f g), f-g,-i(f+g)) .
$$

Here $\psi$ is analytic and the order of the zeros of $\psi$ must be greater than the order of the poles of $f, g$ at each point. If $\psi=2 z$ and $f=f_{1}+i f_{2}$, $g=g_{1}+i g_{2}$ then

$$
\begin{aligned}
X_{x}-i X_{y}= & \Phi(z) \\
= & z(1+f g, i(1-f g), f-g,-i(f+g)) \\
= & \left(\left(1+f_{1} g_{1}-f_{2} g_{2}\right) x-\left(f_{2} g_{1}+f_{1} g_{2}\right) y,\right. \\
& \left(f_{2} g_{1}+f_{1} g_{2}\right) x-y+f_{1} g_{1} y-f_{2} g_{2} y, \\
& \left.\left(f_{1}-g_{1}\right) x+\left(-f_{2}+g_{2}\right) y,\left(f_{2}+g_{2}\right) x+\left(f_{1}+g_{1}\right) y\right) \\
& -i\left(-y-f_{1}\left(g_{2} x+g_{1} y\right)+f_{2}\left(-g_{1} x+g_{2} y\right),\right. \\
& \left(-1+f_{1} g_{1}-f_{2} g_{2}\right) x-\left(f_{2} g_{1}+f_{1} g_{2}\right) y, \\
& +\left(-f_{2}+g_{2}\right) x+\left(-f_{1}+g_{1}\right) y, \\
& \left.\left(f_{1}+g_{1}\right) x-\left(f_{2}+g_{2}\right) y\right)
\end{aligned}
$$

We set

$$
\begin{aligned}
w_{1}= & \left(-\left(f_{2} g_{1} x+f_{1} g_{2} x-y+f_{1} g_{1} y-f_{2} g_{2} y\right),\right. \\
& \left(1+f_{1} g_{1}-f_{2} g_{2}\right) x-\left(f_{2} g_{1}+f_{1} g_{2}\right) y, \\
& -\left(\left(f_{2}+g_{2}\right) x+\left(f_{1}+g_{1}\right) y\right), \\
& \left.\left(f_{1}-g_{1}\right) x+\left(-f_{2}+g_{2}\right) y\right)
\end{aligned}
$$

which is perpendicular to $X_{x}$, and

$$
\begin{aligned}
w_{2}= & \left(-\left(\left(-1+f_{1} g_{1}-f_{2} g_{2}\right) x-\left(f_{2} g_{1}+f_{1} g_{2}\right) y\right),\right. \\
& -y-f_{1}\left(g_{2} x+g_{1} y\right)+f_{2}\left(-g_{1} x+g_{2} y\right), \\
& -\left(f_{1} x+g_{1} x-\left(f_{2}+g_{2}\right) y\right), \\
& \left.-f_{2} x+g_{2} x+\left(-f_{1}+g_{1}\right) y\right)
\end{aligned}
$$

$$
\begin{aligned}
& \sqrt{\frac{a}{a^{2}-b^{2}}}=\sqrt{\frac{1+f_{1}^{2}+f_{2}^{2}}{4\left(f_{1}^{2}+f_{2}^{2}\right)\left(x^{2}+y^{2}\right)\left(g_{1}^{2}+g_{2}^{2}+1\right)}}, \\
& \frac{b}{a}=-\frac{-1+f_{1}^{2}+f_{2}^{2}}{1+f_{1}^{2}+f_{2}^{2}} .
\end{aligned}
$$

Then

which is perpendicular to $X_{y}$.

So far we see that: 
CBÜ F Bil. Dergi., Cilt 13, Say1 1, 2017, 155-163 s

$$
n_{1}=\sqrt{\frac{a}{a^{2}-b^{2}}}\left[\begin{array}{c}
\left(\begin{array}{c}
-\left(f_{1} g_{2}+f_{2} g_{1}\right) x-\left(-1+f_{1} g_{1}-f_{2} g_{2}\right) y \\
\left(1+f_{1} g_{1}-f_{2} g_{2}\right) x-\left(f_{2} g_{1}+f_{1} g_{2}\right) y \\
-\left(f_{2}+g_{2}\right) x-\left(f_{1}+g_{1}\right) y \\
\left(f_{1}-g_{1}\right) x+\left(-f_{2}+g_{2}\right) y
\end{array}\right) \\
+\frac{b}{a}\left(\begin{array}{c}
-\left(f_{1} g_{2}+f_{2} g_{1}\right) x+\left(-1-f_{1} g_{1}-f_{2} g_{2}\right) y \\
\left(-1+f_{1} g_{1}-f_{2} g_{2}\right) x-\left(f_{2} g_{1}+f_{1} g_{2}\right) y \\
\left(-f_{2}+g_{2}\right) x+\left(-f_{1}+g_{1}\right) y \\
\left(f_{1}+g_{1}\right) x-\left(f_{2}+g_{2}\right) y
\end{array}\right)
\end{array}\right]
$$

CBU J. of Sci., Volume 13, Issue 1, 2017, p 155-163

$$
\int \Phi(z) d z=\left(\begin{array}{c}
\frac{z^{2}}{2}+\frac{z^{m+n+2}}{m+n+2} \\
i\left(\frac{z^{2}}{2}-\frac{z^{m+n+2}}{m+n+2}\right) \\
\frac{z^{m+2}}{m+2}-\frac{z^{n+2}}{n+2} \\
-i\left(\frac{z^{m+2}}{m+2}+\frac{z^{n+2}}{n+2}\right)
\end{array}\right) .
$$

We let $z=r e^{i \theta}$ and take the real part

With

$$
x=r \cos (\theta), \quad y=r \sin (\theta),
$$

$f_{1}=r^{m} \cos (m \theta), \quad f_{2}=r^{m} \sin (m \theta)$,

$g_{1}=r^{n} \cos (n \theta), g_{2}=r^{n} \sin (n \theta) \quad$ we have: normals $n_{1}(r, \theta)$ :

$$
\frac{1}{\sqrt{\left(r^{2 m}+1\right)\left(r^{2 n}+1\right)}}\left(\begin{array}{c}
r^{m} \sin (\theta)-r^{n} \sin ((m+n+1) \theta) \\
r^{m} \cos (\theta)+r^{n} \cos ((m+n+1) \theta) \\
-r^{m+n} \sin ((n+1) \theta)-\sin ((m+1) \theta) \\
-r^{m+n} \cos ((n+1) \theta)+\cos ((m+1) \theta)
\end{array}\right)
$$

and $n_{2}(r, \theta)$ :

$$
\frac{1}{\sqrt{\left(r^{2 m}+1\right)\left(r^{2 n}+1\right)}}\left(\begin{array}{c}
r^{m} \cos (\theta)-r^{n} \cos ((m+n+1) \theta) \\
-r^{m} \sin (\theta)-r^{n} \sin ((m+n+1) \theta) \\
-r^{m+n} \cos ((n+1) \theta)-\cos ((m+1) \theta) \\
r^{m+n} \sin ((n+1) \theta)-\sin ((m+1) \theta)
\end{array}\right)
$$

\section{Bour's family of surfaces}

We now choose, in analogy with the surface case, $\psi=2 z, f=z^{m}$ and $g=z^{n}$, with $m \neq n$. This gives:

$\Phi(z)=z\left(1+z^{m+n}, i\left(1-z^{m+n}\right), z^{m}-z^{n},-i\left(z^{m}+z^{n}\right)\right)$.

We integrate to get:

$$
\mathrm{B}_{m, n}(r, \theta)=\left(\begin{array}{c}
\frac{r^{2} \cos (2 \theta)}{2}+\frac{r^{m+n+2} \cos ((m+n+2) \theta)}{m+n+2} \\
-\frac{r^{2} \sin (2 \theta)}{2}+\frac{r^{m+n+2} \sin ((m+n+2) \theta)}{m+n+2} \\
\frac{r^{m+2} \cos ((m+2) \theta)}{m+2}-\frac{r^{n+2} \cos ((n+2) \theta)}{n+2} \\
\frac{r^{m+2} \sin ((m+2) \theta)}{m+2}+\frac{r^{n+2} \sin ((n+2) \theta)}{n+2}
\end{array}\right) .
$$

Example: For $m=2, n=0$, we have $\mathrm{B}_{2,0}(r, \theta)$ :

$$
\left(\begin{array}{c}
\frac{r^{2} \cos (2 \theta)}{2}+\frac{r^{4} \cos (4 \theta)}{4} \\
-\frac{r^{2} \sin (2 \theta)}{2}+\frac{r^{4} \sin (4 \theta)}{4} \\
-\frac{r^{2} \cos (2 \theta)}{2}+\frac{r^{4} \cos (4 \theta)}{4} \\
\frac{r^{2} \sin (2 \theta)}{2}+\frac{r^{4} \sin (4 \theta)}{4}
\end{array}\right)=\left(\begin{array}{c}
x(r, \theta) \\
y(r, \theta) \\
z(r, \theta) \\
w(r, \theta)
\end{array}\right)
$$

and $\mathrm{B}_{2,0}(u, v)$ :

$$
\left(\begin{array}{c}
\frac{1}{2}\left(u^{2}-v^{2}\right)+\frac{1}{4} u^{4}-\frac{3}{2} u^{2} v^{2}+\frac{1}{4} v^{4} \\
-u v+u^{3} v-u v^{3} \\
-\frac{1}{2}\left(u^{2}-v^{2}\right)+\frac{1}{4} u^{4}-\frac{3}{2} u^{2} v^{2}+\frac{1}{4} v^{4} \\
u v+u^{3} v-u v^{3}
\end{array}\right)=\left(\begin{array}{c}
x(u, v) \\
y(u, v) \\
z(u, v) \\
w(u, v)
\end{array}\right)
$$

We want to find normals $n_{1}$ and $n_{2}$ of the Bour's minimal surface

$$
\mathrm{B}_{2,0}(u, v)=(x(u, v), y(u, v), z(u, v), w(u, v)),
$$

and degree of the algebraic Bour minimal surface.

Hence, we find the implicit equations $Q(x, y, z, w)=0$ of $\mathrm{B}_{2,0}(u, v)$ using elimination 
CBÜ F Bil. Dergi., Cilt 13, Sayı 1, 2017, 155-163 s

techniques in the cartesian coordinates $x, y, z, w$ as follow:

$$
\begin{aligned}
& y^{2}+4 x y^{2}+y^{4}-2 y w-8 x y w-4 y^{3} w-3 w^{2} \\
& +4 x w^{2}+6 y^{2} w^{2}-4 y w^{3}+w^{4}
\end{aligned}
$$

and

$$
\begin{aligned}
& -3 y^{2}+y^{4}+4 y^{2} z-2 y w-4 y^{3} w-8 y z w+w^{2} \\
& +6 y^{2} w^{2}+4 z w^{2}-4 y w^{3}+w^{4} .
\end{aligned}
$$

without $z$ and $x$, respectively. But we should get with $x, y, z, w$. On the other hand, we use the Sylvester elimination technique and find the implicit eq. as follows:

$$
\operatorname{det}\left(\begin{array}{cccc}
1 & 0 & A & 0 \\
0 & 1 & 0 & A \\
1 & 0 & B & 0 \\
0 & 1 & 0 & B
\end{array}\right)=(B-A)^{2}
$$

$=\left(2 x+2 z-2 w y+2 x z+w^{2}-x^{2}+y^{2}-z^{2}\right)^{2}$,

where

$$
\begin{gathered}
A=-2(x-z)^{2}+2(x+z), \\
B=-(x-z)^{2}-(w-y)^{2} .
\end{gathered}
$$

For short, taking $r^{4}=t^{2}=k$, then we get

$$
\begin{aligned}
\operatorname{det}\left(\begin{array}{ll}
1 & A \\
1 & B
\end{array}\right) & =B-A \\
& =2 x+2 z-2 w y+2 x z+w^{2}-x^{2}+y^{2}-z^{2} .
\end{aligned}
$$

Hence, the irreducible implicit equation is

$$
Q(x, y, z, w)=2 x+2 z-2 w y+2 x z+w^{2}-x^{2}+y^{2}-z^{2}
$$

with $\operatorname{deg}\left(B_{2,0}\right)=2$. So, $B_{2,0}$ is an algebraic minimal surface in 4-space. Then find $P_{1}$ using
CBU J. of Sci., Volume 13, Issue 1, 2017, p 155-163

$$
x X_{1}+y Y_{1}+z Z_{1}+w W_{1}+P_{1}=0,
$$

where

$$
n_{1}=\left(X_{1}(u, v), Y_{1}(u, v), Z_{1}(u, v), W_{1}(u, v)\right),
$$

and $P_{1}=P_{1}(u, v)$. Similarly, find $P_{2}$ using

$$
x X_{2}+y Y_{2}+z Z_{2}+w W_{2}+P_{2}=0 \text {, }
$$

where

$$
n_{2}=\left(X_{2}(u, v), Y_{2}(u, v), Z_{2}(u, v), W_{2}(u, v)\right),
$$

and $P_{2}=P_{2}(u, v)$. Therefore, inhomogeneous tangential coordinates of the Bour surface, using $n_{1}$ (resp. using $n_{2}$ ), are $a_{1}=X_{1} / P_{1}, b_{1}=Y_{1} / P_{1}$, $c_{1}=Z_{1} / P_{1}, d_{1}=W_{1} / P_{1} \quad$ (resp. $\quad a_{2}=X_{2} / P_{2}$, $\left.b_{2}=Y_{2} / P_{2}, \quad c_{2}=Z_{2} / P_{2}, \quad d_{2}=W_{2} / P_{2}\right)$.

Hence, we can find the implicit eq.

$$
\widetilde{Q_{1}}\left(a_{1}, b_{1}, c_{1}, d_{1}\right)=0
$$

(resp. $\left.\widetilde{Q_{2}}\left(a_{2}, b_{2}, c_{2}, d_{2}\right)=0\right)$

of

$$
\widetilde{\mathfrak{B}}_{2,0}(u, v)
$$

using elimination techniques in the inhomogeneous tangential coordinates $a_{1}, b_{1}, c_{1}, d_{1}$ (resp. $a_{2}, b_{2}, c_{2}, d_{2}$ ) and can find the classes of the algebraic Bour minimal surface (we have 2 normals and then have 2 classes).

$$
\begin{aligned}
\left(n_{1}\right)_{2,0}(r, \theta) & =\frac{1}{\sqrt{2\left(r^{4}+1\right)}}\left(\begin{array}{c}
r^{2} \sin (\theta)-\sin (3 \theta) \\
r^{2} \cos (\theta)+\cos (3 \theta) \\
-r^{2} \sin (\theta)-\sin (3 \theta) \\
-r^{2} \cos (\theta)+\cos (3 \theta)
\end{array}\right) \\
& =\left(\begin{array}{c}
X_{1}(r, \theta) \\
Y_{1}(r, \theta) \\
Z_{1}(r, \theta) \\
W_{1}(r, \theta)
\end{array}\right),
\end{aligned}
$$


CBÜ F Bil. Dergi., Cilt 13, Sayı 1, 2017, 155-163 s and then

$$
\begin{aligned}
& \left(n_{1}\right)_{2,0}(u, v)=\left(\begin{array}{c}
\frac{v\left(u^{4}+2 u^{2} v^{2}-3 u^{2}+v^{4}+v^{2}\right)}{\left(u^{2}+v^{2}\right)^{\frac{3}{2}} \sqrt{2\left(\left(u^{2}+v^{2}\right)^{2}+1\right)}} \\
\frac{u\left(u^{4}+2 u^{2} v^{2}+u^{2}+v^{4}-3 v^{2}\right)}{\left(u^{2}+v^{2}\right)^{\frac{3}{2}} \sqrt{2\left(\left(u^{2}+v^{2}\right)^{2}+1\right)}} \\
-\frac{v\left(u^{4}+2 u^{2} v^{2}+3 u^{2}+v^{4}-v^{2}\right)}{\left(u^{2}+v^{2}\right)^{\frac{3}{2}} \sqrt{2\left(\left(u^{2}+v^{2}\right)^{2}+1\right)}} \\
-\frac{u\left(u^{4}+2 u^{2} v^{2}-u^{2}+v^{4}+3 v^{2}\right)}{\left(u^{2}+v^{2}\right)^{\frac{3}{2}} \sqrt{2\left(\left(u^{2}+v^{2}\right)^{2}+1\right)}}
\end{array}\right) \\
& =\left(\begin{array}{c}
X_{1}(u, v) \\
Y_{1}(u, v) \\
Z_{1}(u, v) \\
W_{1}(u, v)
\end{array}\right) .
\end{aligned}
$$

Using $x X_{1}+y Y_{1}+z Z_{1}+w W_{1}+P_{1}=0$, we get

$$
P_{1}=\frac{v \sqrt{2}\left(\sqrt{u^{2}+v^{2}}\right)^{3}}{4 \sqrt{\left(u^{2}+v^{2}\right)^{2}+1}}
$$

and then

$$
\begin{aligned}
& a_{1}=X_{1} / P_{1}=\frac{2\left(u^{4}+2 u^{2} v^{2}-3 u^{2}+v^{4}+v^{2}\right)}{\left(u^{2}+v^{2}\right)^{3}}, \\
& b_{1}=Y_{1} / P_{1}=\frac{2 u\left(u^{4}+2 u^{2} v^{2}+u^{2}+v^{4}-3 v^{2}\right)}{v\left(u^{2}+v^{2}\right)^{3}}, \\
& c_{1}=Z_{1} / P_{1}=\frac{-2\left(u^{4}+2 u^{2} v^{2}+3 u^{2}+v^{4}-v^{2}\right)}{\left(u^{2}+v^{2}\right)^{3}}, \\
& d_{1}=W_{1} / P_{1}=\frac{-2 u\left(u^{4}+2 u^{2} v^{2}-u^{2}+v^{4}+3 v^{2}\right)}{v\left(u^{2}+v^{2}\right)^{3}} .
\end{aligned}
$$

Hence, in the inhomogeneous tangential coordinates $a_{1}, b_{1}, c_{1}, d_{1}$, parametric eq. of Bour surface is
CBU J. of Sci., Volume 13, Issue 1, 2017, p 155-163

$$
\begin{aligned}
\mathfrak{B}_{2,0}(u, v) & =\frac{2}{v\left(u^{2}+v^{2}\right)^{3}}\left(\begin{array}{c}
v\left(u^{4}+2 u^{2} v^{2}-3 u^{2}+v^{4}+v^{2}\right) \\
u\left(u^{4}+2 u^{2} v^{2}+u^{2}+v^{4}-3 v^{2}\right) \\
-v\left(u^{4}+2 u^{2} v^{2}+3 u^{2}+v^{4}-v^{2}\right) \\
-u\left(u^{4}+2 u^{2} v^{2}-u^{2}+v^{4}+3 v^{2}\right)
\end{array}\right) \\
& =\left(\begin{array}{c}
a_{1}(u, v) \\
b_{1}(u, v) \\
c_{1}(u, v) \\
d_{1}(u, v)
\end{array}\right) .
\end{aligned}
$$

So, we have 6 implicit eqs.

$$
\widetilde{Q}_{1}\left(a_{1}, b_{1}, c_{1}, d_{1}\right)=0
$$

of

$$
\widetilde{\mathfrak{B}}_{2,0}(u, v)
$$

using elimination techniques in the inhomogeneous tangential coordinates $a_{1}, b_{1}, c_{1}, d_{1}$, as follow:

$$
\begin{aligned}
\widetilde{Q}_{1}\left(a_{1}, b_{1}, c_{1}, d_{1}\right)= & -a_{1}^{2} b_{1}+a_{1}^{2} d_{1}+2 a_{1} b_{1} c_{1} \\
& -2 a_{1} c_{1} d_{1}-b_{1} c_{1}^{2}+c_{1}^{2} d_{1} \\
& -4 a_{1} b_{1}+4 c_{1} d_{1}
\end{aligned}
$$

or

$$
\begin{aligned}
\widetilde{Q}_{1}\left(a_{1}, b_{1}, c_{1}, d_{1}\right)= & -a_{1}^{3}+2 a_{1}^{2} c_{1}+2 a_{1} b_{1}^{2} \\
& -2 a_{1} b_{1} d_{1}-a_{1} c_{1}^{2}-b_{1}^{2} c_{1} \\
& +c_{1} d_{1}^{2}+4 a_{1}^{2}+4 a_{1} c_{1} \\
& +4 b_{1} d_{1}+4 d_{1}^{2}
\end{aligned}
$$

or

$$
\begin{aligned}
\widetilde{Q}_{1}\left(a_{1}, b_{1}, c_{1}, d_{1}\right)= & -2 a_{1}^{3}+5 a_{1}^{2} c_{1}+3 a_{1} b_{1}^{2}-4 a_{1} b_{1} d_{1} \\
& -4 a_{1} c_{1}^{2}+a_{1} d_{1}^{2}-2 b_{1}^{2} c_{1}+2 b_{1} c_{1} d_{1} \\
& +c_{1}^{3}+8 a_{1}^{2}+4 a_{1} c_{1}-4 b_{1}^{2}+4 b_{1} d_{1} \\
& -4 c_{1}^{2}+8 d_{1}^{2},
\end{aligned}
$$

or 
CBÜ F Bil. Dergi., Cilt 13, Sayı 1, 2017, 155-163 s

$$
\begin{aligned}
\widetilde{Q}_{1}\left(a_{1}, b_{1}, c_{1}, d_{1}\right)= & a_{1}^{4}-4 a_{1}^{3} c_{1}+6 a_{1}^{2} c_{1}^{2}-4 a_{1} c_{1}^{3} \\
& +c_{1}^{4}-2 a_{1}^{2} c_{1}-2 a_{1} b_{1}^{2}+4 a_{1} b_{1} d_{1} \\
& +4 a_{1} c_{1}^{2}-2 a_{1} d_{1}^{2}-2 c_{1}^{3}-16 a_{1}^{2} \\
& -24 a_{1} c_{1}-8 b_{1}^{2}-24 b_{1} d_{1}-8 c_{1}^{2} \\
& -16 d_{1}^{2},
\end{aligned}
$$

or

$$
\begin{aligned}
\widetilde{Q}_{1}\left(a_{1}, b_{1}, c_{1}, d_{1}\right)= & -2 a_{1}^{3} b_{1}+6 a_{1}^{2} b_{1} c_{1}-a_{1} b_{1}^{3}+3 a_{1} b_{1}^{2} d_{1} \\
& -6 a_{1} b_{1} c_{1}^{2}-3 a_{1} b_{1} d_{1}^{2}+a_{1} d_{1}^{3}+2 b_{1} c_{1}^{3} \\
& -16 a_{1}^{2} b_{1}+16 a_{1}^{2} d_{1}+16 a_{1} b_{1} c_{1}-4 a_{1} c_{1} \\
& -4 b_{1}^{3}-8 b_{1}^{2} d_{1}-12 b_{1} c_{1}^{2}+4 b_{1} d_{1}^{2}+8 d_{1}^{3} \\
& -32 a_{1} b_{1}+32 c_{1} d_{1},
\end{aligned}
$$$$
-16 a_{1}^{2} b_{1}+16 a_{1}^{2} d_{1}+16 a_{1} b_{1} c_{1}-4 a_{1} c_{1} d_{1} \Phi(z)=\left(1+z^{m+n}, i\left(1-z^{m+n}\right), z^{m}-z^{n},-i\left(z^{m}+z^{n}\right)\right) \text {. }
$$

or

$$
\begin{aligned}
\widetilde{Q}_{1}\left(a_{1}, b_{1}, c_{1}, d_{1}\right)= & a_{1} b_{1}^{4}-4 a_{1} b_{1}^{3} d_{1}+6 a_{1} b_{1}^{2} d_{1}^{2}-4 a_{1} b_{1} d_{1}^{3}+a_{1} d_{1}^{4} \\
& -16 a_{1}^{4}+38 a_{1}^{3} c_{1}+14 a_{1}^{2} b_{1}^{2}-44 a_{1}^{2} b_{1} d_{1} \\
& -28 a_{1}^{2} c_{1}^{2}+22 a_{1}^{2} d_{1}^{2}+16 a_{1} b_{1}^{2} c_{1}+6 a_{1} c_{1}^{3} \\
& +4 b_{1}^{4}+4 b_{1}^{3} d_{1}-8 b_{1}^{2} c_{1}^{2}-12 b_{1}^{2} d_{1}^{2}-4 b_{1} d_{1}^{3} \\
& +8 d_{1}^{4}+144 a_{1}^{3}-144 a_{1}^{2} c_{1}-208 a_{1} b_{1}^{2} \\
& +168 a_{1} b_{1} d_{1}+104 a_{1} c_{1}^{2}+40 a_{1} d_{1}^{2}+80 b_{1}^{2} c_{1} \\
& -24 c_{1}^{3}-320 a_{1}^{2}-224 a_{1} c_{1}+96 b_{1}^{2}-224 b_{1} d_{1} \\
& +96 c_{1}^{2}-320 d_{1}^{2} .
\end{aligned}
$$

So,

$$
\operatorname{classes}\left(\widetilde{\mathfrak{B}}_{2,0}\right)=3,4,5
$$

We can use the same techniques for $\left(n_{2}\right)_{2,0}$ :

$$
\begin{aligned}
\left(n_{2}\right)_{2,0}(r, \theta) & =\frac{1}{\sqrt{2\left(r^{4}+1\right)}}\left(\begin{array}{c}
r^{2} \cos (\theta)-\cos (3 \theta) \\
-r^{2} \sin (\theta)-\sin (3 \theta) \\
-r^{2} \cos (\theta)-\cos (3 \theta) \\
r^{2} \sin (\theta)-\sin (3 \theta)
\end{array}\right) \\
& =\left(\begin{array}{c}
X_{2}(r, \theta) \\
Y_{2}(r, \theta) \\
Z_{2}(r, \theta) \\
W_{2}(r, \theta)
\end{array}\right),
\end{aligned}
$$

Example: For $m=2, \quad n=0$, we have $\mathrm{E}_{2,0}(r, \theta)$ :

$$
\left(\begin{array}{c}
\frac{r^{3} \cos (3 \theta)}{3}+r \cos (\theta) \\
\frac{r^{3} \sin (3 \theta)}{3}-r \sin (\theta) \\
\frac{r^{3} \cos (3 \theta)}{3}-r \cos (\theta) \\
\frac{r^{3} \sin (3 \theta)}{3}+r \sin (\theta)
\end{array}\right)=\left(\begin{array}{c}
x(r, \theta) \\
y(r, \theta) \\
z(r, \theta) \\
w(r, \theta)
\end{array}\right)
$$

and $\mathrm{E}_{2,0}(u, v)$ :

$$
\left(n_{2}\right)_{2,0}(u, v)=\left(\begin{array}{c}
X_{2}(u, v) \\
Y_{2}(u, v) \\
Z_{2}(u, v) \\
W_{2}(u, v)
\end{array}\right)
$$

\section{Enneper's family of surfaces}

We now choose, in analogy with the surface case, $\psi=2, f=z^{m}$ and $g=z^{n}$, with $m \neq n$. This gives:

We integrate to get:

$$
\int \Phi(z) d z=\left(\begin{array}{c}
z+\frac{z^{m+n+1}}{m+n+1} \\
i\left(z-\frac{z^{m+n+1}}{m+n+1}\right) \\
\frac{z^{m+1}}{m+1}-\frac{z^{n+1}}{n+1} \\
-i\left(\frac{z^{m+1}}{m+1}+\frac{z^{n+1}}{n+1}\right)
\end{array}\right)
$$

We let $z=r e^{i \theta}$ and take the real part

$$
\mathrm{E}_{m, n}(r, \theta)=\left(\begin{array}{c}
r \cos (\theta)+\frac{r^{m+n+1} \cos ((m+n+1) \theta)}{m+n+1} \\
-r \sin (\theta)+\frac{r^{m+n+1} \sin ((m+n+1) \theta)}{m+n+1} \\
\frac{r^{m+1} \cos ((m+1) \theta)}{m+1}-\frac{r^{n+1} \cos ((n+1) \theta)}{n+1} \\
\frac{r^{m+1} \sin ((m+1) \theta)}{m+1}+\frac{r^{n+1} \sin ((n+1) \theta)}{n+1}
\end{array}\right) .
$$


CBÜ F Bil. Dergi., Cilt 13, Sayı 1, 2017, 155-163 s

$$
\left(\begin{array}{c}
\frac{1}{3} u^{3}-u v^{2}+u \\
u^{2} v-\frac{1}{3} v^{3}-v \\
\frac{1}{3} u^{3}-u v^{2}-u \\
u^{2} v-\frac{1}{3} v^{3}+v
\end{array}\right)=\left(\begin{array}{c}
x(u, v) \\
y(u, v) \\
z(u, v) \\
w(u, v)
\end{array}\right)
$$

where $u=r \cos \theta, \quad v=r \sin \theta$.

We want to find normals $n_{1}$ and $n_{2}$ of the Enneper's minimal surface

$$
\mathrm{E}_{2,0}(u, v)=(x(u, v), y(u, v), z(u, v), w(u, v)),
$$

and degree of the algebraic Enneper minimal surface.

We have $r+A=0, r^{3}+B=0$ and

$$
\operatorname{Syl}(A, B, r)=\operatorname{det}\left(\begin{array}{cccc}
1 & A & 0 & 0 \\
0 & 1 & A & 0 \\
0 & 0 & 1 & A \\
1 & 0 & 0 & B
\end{array}\right)=B-A^{3},
$$

where

$$
\begin{aligned}
& A=-\frac{1}{4}\left((x-z)^{2}+(w-y)^{2}\right), \\
& B=-\frac{9}{4}\left((x+z)^{2}+(w+y)^{2}\right) .
\end{aligned}
$$

Hence, we find the irreducible implicit equation $Q(x, y, z, w)=0$ of $\mathrm{E}_{2,0}(u, v)$ using elimination techniques in the cartesian coordinates $x, y, z, w$ as follows:
CBU J. of Sci., Volume 13, Issue 1, 2017, p 155-163

$$
\begin{aligned}
& w^{6}-6 w^{5} y+3 w^{4} x^{2}-6 w^{4} x z+15 w^{4} y^{2}+3 w^{4} z^{2} \\
& -12 w^{3} x^{2} y+24 w^{3} x y z-20 w^{3} y^{3}-12 w^{3} y z^{2}+3 w^{2} x^{4} \\
& -12 w^{2} x^{3} z+18 w^{2} x^{2} y^{2}+18 w^{2} x^{2} z^{2}-36 w^{2} x y^{2} z \\
& -12 w^{2} x z^{3}+15 w^{2} y^{4}+18 w^{2} y^{2} z^{2}+3 w^{2} z^{4}-144 w^{2} \\
& -6 w x^{4} y+24 w x^{3} y z-12 w x^{2} y^{3}-36 w x^{2} y z^{2}+24 w x y^{3} z \\
& +24 w x y z^{3}-6 w y^{5}-12 w y^{3} z^{2}-6 w y z^{4}-288 w y+x^{6} \\
& -6 x^{5} z+3 x^{4} y^{2}+15 x^{4} z^{2}-12 x^{3} y^{2} z-20 x^{3} z^{3}+3 x^{2} y^{4} \\
& +18 x^{2} y^{2} z^{2}+15 x^{2} z^{4}-144 x^{2}-6 x y^{4} z-12 x y^{2} z^{3}-6 x z^{5} \\
& -288 x z+y^{6}+3 y^{4} z^{2}+3 y^{2} z^{4}-144 y^{2}+z^{6}-144 z^{2} .
\end{aligned}
$$

Its degree is $\operatorname{deg}\left(\mathrm{E}_{2,0}\right)=6$. So, $Q(x, y, z, w)=0$ is an implicit algebraic Enneper type minimal surface in 4-space. Then find $P_{1}$ using

$$
x X_{1}+y Y_{1}+z Z_{1}+w W_{1}+P_{1}=0,
$$

where

$$
n_{1}=\left(X_{1}(u, v), Y_{1}(u, v), Z_{1}(u, v), W_{1}(u, v)\right)
$$

and $P_{1}=P_{1}(u, v)$. Similarly, find $P_{2}$ using

$$
x X_{2}+y Y_{2}+z Z_{2}+w W_{2}+P_{2}=0,
$$

where

$$
n_{2}=\left(X_{2}(u, v), Y_{2}(u, v), Z_{2}(u, v), W_{2}(u, v)\right),
$$

and $P_{2}=P_{2}(u, v)$. Therefore, inhomogeneous tangential coordinates of the Enneper surface, using $n_{1}$ (resp. using $n_{2}$ ), are $a_{1}=X_{1} / P_{1}, b_{1}=Y_{1} / P_{1}$, $c_{1}=Z_{1} / P_{1}, \quad d_{1}=W_{1} / P_{1} \quad$ (resp. $a_{2}=X_{2} / P_{2}$, $\left.b_{2}=Y_{2} / P_{2}, \quad c_{2}=Z_{2} / P_{2}, \quad d_{2}=W_{2} / P_{2}\right)$.

Hence, we can find the implicit eq.

$$
\widetilde{Q_{1}}\left(a_{1}, b_{1}, c_{1}, d_{1}\right)=0
$$

(resp. $\left.\widetilde{Q_{2}}\left(a_{2}, b_{2}, c_{2}, d_{2}\right)=0\right)$

of

$$
\widetilde{\mathfrak{E}}_{2,0}(u, v)
$$


CBÜ F Bil. Dergi., Cilt 13, Sayı 1, 2017, 155-163 s

using elimination techniques in the inhomogeneous tangential coordinates $a_{1}, b_{1}, c_{1}, d_{1}$ (resp. $\left.a_{2}, b_{2}, c_{2}, d_{2}\right)$ and can find the classes of the algebraic Enneper minimal surface (we have 2 normals and then have 2 classes). $\left(n_{1}\right)_{2,0}(r, \theta)$ is as follows:

$$
\begin{aligned}
\left(n_{1}\right)_{2,0}(r, \theta) & =\frac{1}{\sqrt{2\left(r^{4}+1\right)}}\left(\begin{array}{c}
-\sin (2 \theta) \\
r^{2}+\cos (2 \theta) \\
-\sin (2 \theta) \\
-r^{2}+\cos (2 \theta)
\end{array}\right) \\
& =\left(\begin{array}{c}
X_{1}(r, \theta) \\
Y_{1}(r, \theta) \\
Z_{1}(r, \theta) \\
W_{1}(r, \theta)
\end{array}\right),
\end{aligned}
$$

and $\left(n_{1}\right)_{2,0}(u, v)$ is as follows:

$$
\begin{aligned}
& \frac{1}{\left(u^{2}+v^{2}\right) \sqrt{2\left(\left(u^{2}+v^{2}\right)^{2}+1\right)}}\left(\begin{array}{c}
-2 u v \\
\left(u^{2}+v^{2}\right)^{2}+\left(u^{2}-v^{2}\right) \\
-2 u v \\
\left(u^{2}-v^{2}\right)-\left(u^{2}+v^{2}\right)^{2}
\end{array}\right) \\
& =\left(\begin{array}{c}
X_{1}(u, v) \\
Y_{1}(u, v) \\
Z_{1}(u, v) \\
W_{1}(u, v)
\end{array}\right) .
\end{aligned}
$$

Using $x X_{1}+y Y_{1}+z Z_{1}+w W_{1}+P_{1}=0$, we get

$$
P_{1}=\frac{2 \sqrt{2} v\left(u^{2}+v^{2}\right)}{3 \sqrt{\left(u^{2}+v^{2}\right)^{2}+1}}
$$

and then

$$
\begin{gathered}
a_{1}=X_{1} / P_{1}=-\frac{3 u}{2\left(u^{2}+v^{2}\right)^{2}}, \\
b_{1}=Y_{1} / P_{1}=\frac{3\left(\left(u^{2}+v^{2}\right)^{2}+u^{2}-v^{2}\right)}{4 v\left(u^{2}+v^{2}\right)^{2}},
\end{gathered}
$$

CBU J. of Sci., Volume 13, Issue 1, 2017, p 155-163

$$
c_{1}=Z_{1} / P_{1}=-\frac{3 u}{2\left(u^{2}+v^{2}\right)^{2}},
$$

$$
d_{1}=W_{1} / P_{1}=-\frac{3\left(\left(u^{2}+v^{2}\right)^{2}+v^{2}-u^{2}\right)}{4 v\left(u^{2}+v^{2}\right)^{2}} .
$$

Hence, using $\mathrm{E}_{2,0}(u, v)$ and $\left(n_{1}\right)_{2,0}(u, v)$, we get the first parametric eq. of Enneper type surface

$$
\mathfrak{E}_{2,0}(u, v)
$$

in the inhomogeneous tangential coordinates $a_{1}, b_{1}, c_{1}, d_{1}$ as follows:

$$
\begin{aligned}
\widetilde{\mathfrak{E}}_{2,0}(u, v) & =\frac{3}{4 v\left(u^{2}+v^{2}\right)^{2}}\left(\begin{array}{c}
-2 u v \\
\left(u^{2}+v^{2}\right)^{2}+u^{2}-v^{2} \\
-2 u v \\
\left(u^{2}+v^{2}\right)^{2}+v^{2}-u^{2}
\end{array}\right) \\
& =\left(\begin{array}{c}
a_{1}(u, v) \\
b_{1}(u, v) \\
c_{1}(u, v) \\
d_{1}(u, v)
\end{array}\right) .
\end{aligned}
$$

Then we have implicit eq.

$$
\widetilde{Q}_{1}\left(a_{1}, b_{1}, c_{1}, d_{1}\right)=0
$$

of the first surface

$$
\widetilde{\mathfrak{E}}_{2,0}(u, v)
$$

using elimination techniques in the inhomogeneous tangential coordinates $a_{1}, b_{1}, c_{1}, d_{1}$, as follows:

$$
\begin{aligned}
& \widetilde{Q}_{1}\left(a_{1}, b_{1}, c_{1}, d_{1}\right)=16 a_{1}^{2} b_{1}^{6}-96 a_{1}^{2} b_{1}^{5} d_{1}+240 a_{1}^{2} b_{1}^{4} d_{1}^{2} \\
& -320 a_{1}^{2} b_{1}^{3} c_{1}^{3}+240 a_{1}^{2} b_{1}^{2} d_{1}^{4}-96 a_{1}^{2} b_{1} d_{1}^{5}+16 a_{1}^{2} d_{1}^{6} \\
& -144 a_{1}^{2} b_{1}^{4}+288 a_{1}^{2} b_{1}^{3} d_{1}-288 a_{1}^{2} b_{1} d_{1}^{3}+144 a_{1}^{2} d_{1}^{4}-36 b_{1}^{6} \\
& +108 b_{1}^{4} d_{1}^{2}-108 b_{1}^{2} d_{1}^{4}+36 d_{1}^{6}-1296 a_{1}^{4}-648 a_{1}^{2} b_{1}^{2} \\
& -1296 a_{1}^{2} b_{1} d_{1}-648 a_{1}^{2} d_{1}^{2}-81 b_{1}^{4}-324 b_{1}^{3} d_{1}-486 b_{1}^{2} d_{1}^{2} \\
& -324 b_{1} d_{1}^{3}-81 d_{1}^{4} .
\end{aligned}
$$

So,

$$
\operatorname{class}\left(\widetilde{\mathfrak{E}}_{2,0}\right)=8 .
$$


CBÜ F Bil. Dergi., Cilt 13, Sayı 1, 2017, 155-163 s

We can use the same techniques for

$$
\begin{aligned}
\left(n_{2}\right)_{2,0}(r, \theta) & =\frac{1}{\sqrt{2 r^{4}\left(r^{4}+1\right)}}\left(\begin{array}{c}
r^{4}-r^{2} \cos (2 \theta) \\
-r^{2} \sin (2 \theta) \\
-r^{4}-r^{2} \cos (2 \theta) \\
-r^{2} \sin (2 \theta)
\end{array}\right) \\
& =\left(\begin{array}{c}
X_{2}(r, \theta) \\
Y_{2}(r, \theta) \\
Z_{2}(r, \theta) \\
W_{2}(r, \theta)
\end{array}\right),
\end{aligned}
$$

and $\left(n_{2}\right)_{2,0}(u, v)$ :

$$
\begin{aligned}
& \frac{1}{\left(u^{2}+v^{2}\right) \sqrt{2\left(\left(u^{2}+v^{2}\right)^{2}+1\right)}}\left(\begin{array}{c}
\left(u^{2}+v^{2}\right)^{2}+v^{2}-u^{2} \\
-2 u v \\
-\left(u^{2}+v^{2}\right)^{2}+v^{2}-u^{2} \\
-2 u v
\end{array}\right) \\
& =\left(\begin{array}{c}
X_{2}(u, v) \\
Y_{2}(u, v) \\
Z_{2}(u, v) \\
W_{2}(u, v)
\end{array}\right) .
\end{aligned}
$$

Using $\mathrm{E}_{2,0}(u, v)$ and $\left(n_{2}\right)_{2,0}(u, v)$, we get

$$
P_{2}=-\frac{2 \sqrt{2} u\left(u^{2}+v^{2}\right)}{3 \sqrt{\left(u^{2}+v^{2}\right)^{2}+1}}
$$

Hence, we obtain the second surface:

$$
\begin{aligned}
\widetilde{\mathfrak{E}}_{2,0}(u, v) & =\frac{3}{4 v\left(u^{2}+v^{2}\right)^{2}}\left(\begin{array}{c}
-\left[\left(u^{2}+v^{2}\right)^{2}+v^{2}-u^{2}\right] \\
2 u v \\
-\left[\left(u^{2}+v^{2}\right)^{2}+u^{2}-v^{2}\right] \\
2 u v
\end{array}\right) \\
& =\left(\begin{array}{c}
a_{2}(u, v) \\
b_{2}(u, v) \\
c_{2}(u, v) \\
d_{2}(u, v)
\end{array}\right) .
\end{aligned}
$$

CBU J. of Sci., Volume 13, Issue 1, 2017, p 155-163 So, we have implicit eq.

$$
\widetilde{Q}_{2}\left(a_{2}, b_{2}, c_{2}, d_{2}\right)=0
$$

of the second surface

$$
\widetilde{\mathfrak{E}}_{2,0}(u, v)
$$

using elimination techniques in the inhomogeneous tangential coordinates $a_{2}, b_{2}, c_{2}, d_{2}$ as follows:

$$
\begin{aligned}
& \widetilde{Q}_{2}\left(a_{2}, b_{2}, c_{2}, d_{2}\right)=16 a_{2}^{6} b_{2}^{2}-96 a_{2}^{5} b_{2}^{2} c_{2}+240 a_{2}^{4} b_{2}^{2} c_{2}^{2} \\
& -320 a_{2}^{3} b_{2}^{2} c_{2}^{3}+240 a_{2}^{2} b_{2}^{2} c_{2}^{4}-96 a_{2} b_{2}^{2} c_{2}^{5}+16 b_{2}^{2} c_{2}^{6} \\
& -36 a_{2}^{6}-144 a_{2}^{4} b_{2}^{2}+108 a_{2}^{4} c_{2}^{2}+288 a_{2}^{3} b_{2}^{2} c_{2}-108 a_{2}^{2} c_{2}^{4} \\
& -288 a_{2} b_{2}^{2} c_{2}^{3}+144 b_{2}^{2} c_{2}^{4}+36 c_{2}^{6}-81 a_{2}^{4}-324 a_{2}^{3} c_{2} \\
& -648 a_{2}^{2} b_{2}^{2}-486 a_{2}^{2} c_{2}^{2}-1296 a_{2} b_{2}^{2} c_{2}-324 a_{2} c_{2}^{3} \\
& -1296 b_{2}^{4}-648 b_{2}^{2} c_{2}^{2}-81 c_{2}^{4} .
\end{aligned}
$$

Then we have

$$
\operatorname{class}\left(\widetilde{\mathfrak{E}}_{2,0}\right)=8 .
$$

\section{References}

[1] Eisenhart, L.P. A Treatise on the Differential Geometry of Curves and Surfaces, Dover Publications, N.Y. 1909.

[2] Ganchev, G.; Milousheva, V. An invariant theory of surfaces in the four-dimensional Euclidean or Minkowski space. Pliska Stud. Math. Bulgar. 2012; 21, 177--200.

[3] Hoffman, D.A.; Osserman, R. The Geometry of the Generalized Gauss Map. Memoirs of the AMS, 1980.

[4] Moore, C. Surfaces of rotation in a space of four dimensions. The Annals of Math., 2nd Ser., 1919; 21(2), 81--93. 